\title{
Pressure and temperature dependence of the zero-field splitting in the ground state of NV centers in diamond: A first-principles study
}

Viktor Ivády, Tamas Simon, Jeronimo R. Maze, Igor Abrikosov and Adam Gali

\author{
Linköping University Post Print
}

Tweet

N.B.: When citing this work, cite the original article.

Original Publication:

Viktor Ivády, Tamas Simon, Jeronimo R. Maze, Igor Abrikosov and Adam Gali, Pressure and temperature dependence of the zero-field splitting in the ground state of NV centers in diamond: A first-principles study, 2014, Physical Review B. Condensed Matter and Materials Physics, (90), 23, 235205.

http://dx.doi.org/10.1103/PhysRevB.90.235205

Copyright: American Physical Society http://www.aps.org/

Postprint available at: Linköping University Electronic Press http://urn.kb.se/resolve?urn=urn:nbn:se:liu:diva-113573 


\title{
Pressure and temperature dependence of the zero-field splitting in the ground state of NV centers in diamond: A first-principles study
}

\author{
Viktor Ivády, ${ }^{1,2}$ Tamás Simon, ${ }^{3}$ Jeronimo R. Maze, ${ }^{4}$ I. A. Abrikosov, ${ }^{1,5,6}$ and Adam Gali ${ }^{3,2, *}$ \\ ${ }^{1}$ Department of Physics, Chemistry and Biology, Linköping University, SE-581 83 Linköping, Sweden \\ ${ }^{2}$ Wigner Research Center for Physics, Institute for Solid State Physics and Optics, Hungarian Academy of Sciences, Budapest, POB 49, \\ H-1525, Hungary \\ ${ }^{3}$ Department of Atomic Physics, Budapest University of Technology and Economics, Budafoki út 8., H-1111, Budapest, Hungary \\ ${ }^{4}$ Facultad de Física, Pontificia Universidad Católica de Chile, Casilla 306, Santiago, Chile \\ ${ }^{5}$ Materials Modeling and Development Laboratory, National University of Science and Technology 'MISIS', 119049 Moscow, Russia \\ ${ }^{6}$ LOCOMAS Laboratory, Tomsk State University, 634050 Tomsk, Russia
}

(Received 7 April 2014; revised manuscript received 19 October 2014; published 19 December 2014)

\begin{abstract}
Nitrogen-vacancy centers in diamond (NV) attract great attention because they serve as a tool in many important applications. The NV center has a polarizable spin $S=1$ ground state and its spin state can be addressed by optically detected magnetic resonance (ODMR) techniques. The $m_{S}=0$ and $m_{S}= \pm 1$ spin levels of the ground state are separated by about $2.88 \mathrm{GHz}$ in the absence of an external magnetic field or any other perturbations. This zero-field splitting (ZFS) can be probed by ODMR. As this splitting changes as a function of pressure and temperature, the NV center might be employed as a sensor operating at the nanoscale. Therefore, it is of high importance to understand the intricate details of the pressure and temperature dependence of this splitting. Here we present an ab initio theory of the ZFS of the NV center as a function of external pressure and temperature including detailed analysis on the contributions of macroscopic and microscopic effects. We found that the pressure dependence is governed by the change in the distance between spins as a consequence of the global compression and the additional local structural relaxation. The local structural relaxation contributes to the change of ZFS with the same magnitude as the global compression. In the case of temperature dependence of ZFS, we investigated the effect of macroscopic thermal expansion as well as the consequent change of the microscopic equilibrium positions. We could conclude that theses effects are responsible for about $15 \%$ of the observed decrease of ZFS.
\end{abstract}

DOI: 10.1103/PhysRevB.90.235205

PACS number(s): 71.15.Mb, 71.55.Ht, 61.72.Bb

\section{INTRODUCTION}

The negatively charged nitrogen-vacancy defect in diamond $[1,2]$, briefly the NV center, is a color center in diamond that is potentially interesting for a large number of applications, ranging from high spatial resolution magnetometry [3-9] through in vivo cellular biomarker [10] to optically addressable solid state qubits [11-13]. The most valuable feature of the NV center is that its spin state can be optically initialized and read-out using optically detected magnetic resonance (ODMR) [13], even at room temperature or above [14-16]. The center has a triplet ground state $(S=1)$, and the $m_{S}=0$ and $m_{S}= \pm 1$ states are separated by the zero-field splitting parameter $(D)$ [17], which is predominantly due to spin-spin interactions between the electrons of the carbon atoms next to the vacancy $[18,19]$. No consensus has been reached about the position of the energy levels of the NV center [20-24], nevertheless, a well-accepted schematic diagram can be given as shown in Fig. 1 where the NV center is depicted in Fig. 2.

The initialization and read-out of the spin is done using a focused laser beam with an appropriate wavelength where even single NV centers can be detected optically at room temperature [25]. Thanks to the intersystem crossing (ISC), an electron excited to the $m_{s}= \pm 1$ state can nonradiatively cascadically decay to the $m_{s}=0$ subspace of the ground state, while an electron excited to the $m_{s}=0$ state can only decay to first order to the $m_{s}=0$ state with the emission

\footnotetext{
*gali.adam@wigner.mta.hu
}

of a photon. Due to this mechanism, after ample time of laser illumination, the defect spin state becomes polarized. If a transversal oscillating magnetic field is applied with a frequency equal to the zero-field splitting parameter $D=$ $2.88 \mathrm{GHz}$, the initialized $m_{s}=0$ spin state can be flipped to the $m_{s}= \pm 1$ subspace. With this flip, the nonradiative path becomes available and thus decreasing the fluorescence by $30 \%$. While a detailed understanding of the nonradiative decay is still missing, it can be suspected that vibrations play an important role in the ISC.

It has been reported lately that the zero-field splitting in the ground state of the nitrogen-vacancy center exhibits a temperature dependence [14]. Motivated by this study several research groups started to apply NV centers as a sensitive thermometer at the nanoscale [26-28]. Recently the range of applications has been extended by the determination of the hydrostatic pressure dependence of the ground state zero-field splitting of the center [29]. To create reliable and robust sensing devices, detailed understanding of the response of the spin resonance of the NV center to temperature and pressure perturbations is crucially needed. Theoretical models, which are described and discussed in the later sections, have already been suggested to explain the experimental observations $[14,15,29,30]$. On the other hand, first-principles simulations serve as another useful tool to gain deeper insight into the physical phenomena of point defects. Although their necessity was pointed out in the literature previously $[29,30]$, such calculations have not been utilized for the NV center yet. 


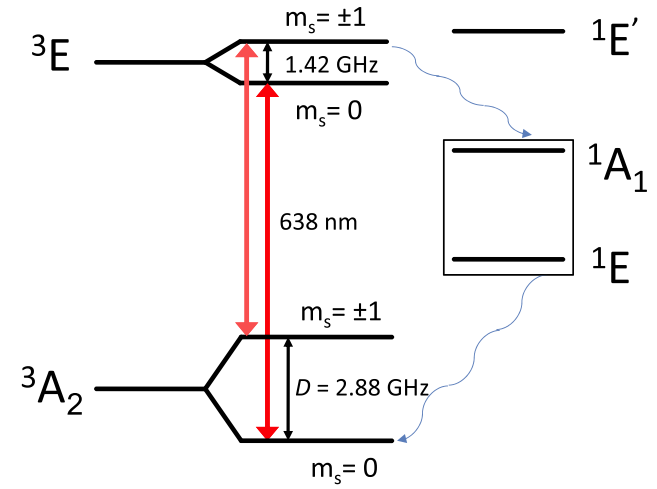

FIG. 1. (Color online) Schematic diagram about energy levels of the NV center at room temperature.

In this paper we study the pressure and temperature dependence of the zero-field splitting tensor of the NV center by means of $a b$ initio supercell calculations. While direct measurement of the movement of the atoms around the defect is not possible, these simulations can provide valuable insight to the microscopic properties of the defect.

The paper is organized as follows. We describe the methods and the implementation of the zero-field splitting tensor calculation in Sec. II. We present and discuss the results in Sec. III. Finally, we summarize our findings in Sec. IV. We provide a brief Appendix to complement our paper.

\section{METHODOLOGY}

In this section we present the $a b$ initio computational methods that are used to obtain the ground state properties and the Kohn-Sham orbitals. Then first-principles calculation of the electron spin-electron spin interaction and its standalone implementation are summarized. (a)

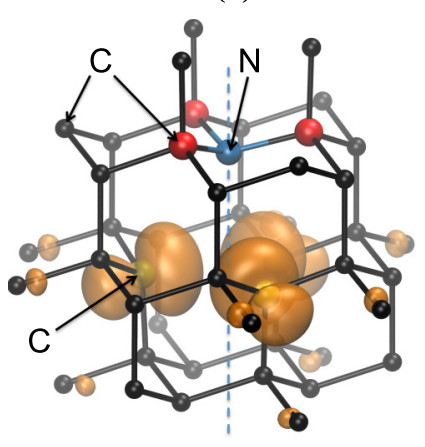

(b)

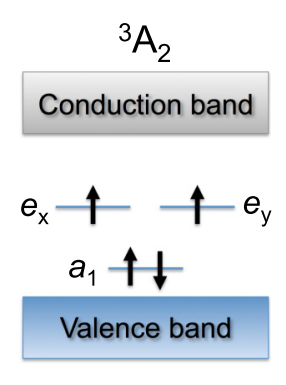

FIG. 2. (Color online) Spin density and electronic structure of the nitrogen-vacancy center in diamond. (a) The orange (light gray) lobes show the spin density in the ${ }^{3} A_{2}$ ground state on the NV center obtained by $a b$ initio DFT calculations. The spin density is mostly originated from the dangling bonds of the carbon atoms at the vacancy site. The symmetry axis is represented with the dashed line. (b) Schematic diagram of the electronic structure where $e_{X}$ and $e_{Y}$ orbitals are localized on the carbon dangling bonds.

\section{A. First-principles calculations}

We applied plane wave supercell density functional theory (DFT) calculations in order to obtain the ground state properties of the NV center. We kept the $C_{3 v}$ symmetry of the defect and $S=1$ spin state. The core electrons of carbon and nitrogen atoms were eliminated by projector augmented-wave (PAW) potentials [31] as implemented in the Vienna ab initio simulation package [32]. To achieve convergent results for the zero-field splitting tensor (ZFST) calculations an increased number of basis functions and accurate atomic positions are needed. For the description of the valence states we used a plane wave basis set to $700 \mathrm{eV}$. The total energy of NV defect was calculated as the function of the coordinates of the atoms in the supercell. The ground state of the defect was found when the calculated total energy showed a global minimum that was achieved by a conjugate gradient algorithm. We applied a very stringent criterion in the geometry optimization procedure: the maximum force acting on the atoms was less than $10^{-4} \mathrm{eV} / \AA$. In order to minimize the spurious self-interaction of the electron spins due to the periodic boundary condition, we utilized 512-atom supercells with $\Gamma$-point sampling of the Brillouin zone. The Perdew-Burke-Ernzerhof (PBE) functional [33] provides superior results on the spin density distribution of the NV center over those obtained by LDA [34,35], therefore we applied a PBE exchange-correlation functional in the evaluation of the ZFST.

\section{B. Calculation of the zero-field splitting tensor}

Sensing applications using the NV center rest upon the precise measurement of the zero-field splitting, thus it is crucial to understand its response to the variation of thermodynamic quantities, such as pressure and temperature. To first order, the zero-field splitting in the ground state is due to the electron spin-spin dipole interaction,

$$
H_{s s}=-\frac{\mu_{0}}{4 \pi} \frac{g^{2} \beta^{2}}{r^{5}}\left[3\left(\mathbf{s}_{1} \cdot \mathbf{r}\right)\left(\mathbf{s}_{2} \cdot \mathbf{r}\right)-\left(\mathbf{s}_{1} \cdot \mathbf{s}_{2}\right) r^{2}\right],
$$

where $\mathbf{r}=\mathbf{r}_{2}-\mathbf{r}_{1}$ is the distance between the spins, $r=|\mathbf{r}|$, $\mathbf{s}_{i}=\frac{1}{2}\left[\sigma_{x}, \sigma_{y}, \sigma_{z}\right]$ is the spin operator vector of particle $i$ where $\sigma_{j}(j=x, y, z)$ are the Pauli matrices, $\beta$ is the Bohr magneton, $g$ is the Landé factor for an electron, and $\mu_{0}$ is the magnetic permeability of vacuum. The spatial and spin dependence can be separated by introducing the total spin operator $\mathbf{S}=\sum_{i} \mathbf{s}_{i}$,

$$
H_{s S}=\mathbf{S}^{\mathrm{T}} \hat{\mathbf{D}} \mathbf{S},
$$

where $\hat{\mathbf{D}}$ is the zero-field splitting tensor (ZFST). In the eigenvalue framework the $3 \times 3$ matrix is diagonal and the spin-spin Hamiltonian is written as

$$
\begin{aligned}
H_{s s} & =D_{x x} S_{x}^{2}+D_{y y} S_{y}^{2}+D_{z z} S_{z}^{2} \\
& =D\left(S_{z}^{2}-\frac{\mathbf{S}^{2}}{3}\right)+\frac{E\left(S_{+}^{2}+S_{-}^{2}\right)}{2},
\end{aligned}
$$

where $S_{i}$ is the $i$ th component of $\mathbf{S}, S^{2}=S_{x}^{2}+S_{y}^{2}+S_{z}^{2}$, $S_{ \pm}=S_{x} \pm i S_{y}$ are the spin raising and lowering operators, $D_{i j}$ are the components of the ZFST, and $D$ and $E$ are the two parameters of the ZFST in the eigenvalue framework. These 
parameters can be expressed by the diagonal elements of the tensor,

$$
D=\frac{3}{2} D_{z z} \text { and } E=\frac{D_{y y}-D_{x x}}{2} .
$$

For $C_{3 v}$ symmetry, the spin Hamiltonian of the ground state is given by

$$
H_{s s}=D\left(S_{z}^{2}-2 / 3\right),
$$

where the quantization axis $z$ of the spin is aligned along the $C_{3}$ axis of the defect and $D$ is the zero-field constant defined in Eq. (4) and shown in Fig. 1.

The matrix elements of the ZFST are calculated from the two-particle density $\varrho=\left|\Psi\left(\mathbf{r}_{1}, \mathbf{r}_{2}\right)\right|$, which can be approximately reproduced by the Kohn-Sham (KS) single determinant wave function of the system. Under this approximation the matrix elements of the ZFST are

$$
D_{a b}=\mu_{0} g_{e}^{2} \beta^{2} \sum_{i<j}^{p+q} \chi_{i j}\left\langle\Psi_{i j}\left(\mathbf{r}_{1}, \mathbf{r}_{2}\right)\left|\frac{r^{2} \delta_{a b}-3 r_{a} r_{b}}{r^{5}}\right| \Psi_{i j}\left(\mathbf{r}_{1}, \mathbf{r}_{2}\right)\right\rangle,
$$

where the sum includes every pair of KS states, i.e., $p$ and $q$ are the number of electrons in the spin-up and spin-down channels, respectively, $\chi_{i j}$ is +1 for parallel spins and -1 for antiparallel spins, $\Psi_{i j}\left(\mathbf{r}_{1}, \mathbf{r}_{2}\right)$ is the Slater determinant of $\psi_{i}$ and $\psi_{j} \mathrm{KS}$ orbitals, and $r_{a}$ is the component of the vector $\mathbf{r}_{2}-\mathbf{r}_{1}$ in the Cartesian coordinate system.

Following this pathway we implemented a standalone code that is capable to read the resultant wave function of a firstprinciples DFT simulation and calculate the matrix elements of the ZFST. This implementation uses the suggestions of Rayson et al. [36] for the case of single $\Gamma$-point sampling of the Brillouin zone. Strictly speaking, the application of this strategy is straightforward with the use of norm-conserving pseudopotentials in the DFT calculations. However, the VASP package, used in our simulations, is a PAW potential based implementation where the PAW potentials have a contribution to the single particle wave functions in the core regions and "only" the pseudo part of the orbitals is resolved in the plane wave basis set. The implementation of full PAW treatment of ZFST is not straightforward [37]. Instead, we applied another strategy here. According to our investigation the contribution of the PAW sphere is about $5 \%$ as $\int\left|\psi_{i \text {,pseudo }}^{\mathrm{KS}}\right|^{2} d^{3} \mathbf{r} \approx 0.95$. In our ZFST calculations we used the normalized pseudo single particle wave functions in Eq. (6). This approximation results in a certain shift in the value of the zero-field splitting. However, we expect that the external perturbations affect to first order the bonding region of the single particle wave functions which is described by the pseudo part. Therefore, we believe that the dependence of the ZFST on the thermodynamic quantities can be well reproduced in our calculations.

\section{RESULTS AND DISCUSSION}

We calculated the pressure and temperature dependence of the zero-field splitting tensor from first principles. In the former case, our results are in good agreement with the experimental observations. Additionally, we could examine different effects and their roles in the perturbation of the ground state splitting. According to our results, the pressure dependence is governed by the change of distances of the electron spins that is a consequence of the global compression and additional local structural relaxation. In the case of temperature dependence of the ZFS, we investigated the effect of a macroscopic thermal expansion as well as the change of the microscopic equilibrium positions. We could conclude that theses effects accounted for about $15 \%$ of the observed decrease of the ZFS, which is in agreement with other thermal expansion models. In the following sections we present the results and discuss them in detail.

\section{A. Pressure dependence of zero-field splitting}

In order to simulate the pressure dependence of the zero-field splitting tensor $D(P)$ from first principles and to examine macroscopic and microscopic effects responsible for the variation of the splitting, we calculated the ZFS in the ground state of the NV center in a 512-atom supercell under external pressure ranging from $P_{\min }=-20 \mathrm{GPa}$ to $P_{\max }=210 \mathrm{GPa}$. The pressure can be simply modeled by varying the size of the supercell. In accordance with the PT-phase diagram of pure carbon, the diamond structure is stable at least up to $1000 \mathrm{GPa}$, therefore the upper limit of the studied pressure interval is still realistic. We expanded our investigation into the negative region as well, in order to further justify the validity of the observed features. In this section all the calculations correspond to $T=0 \mathrm{~K}$, i.e., $D(P, T=0)$ is investigated.

The results of the calculations and a linear fit are shown in Fig. 3. As can be seen, the calculated ZFS linearly depends on the pressure to first order, however, it has a slight curvature on the examined large interval. The observed weak nonlinearity can be explained by understanding the microscopic origin of the pressure dependence that is presented later in this section. There is a recent experimental measurement on the pressure dependence at room temperature carried out by Doherty et al. [29]. They reported linear dependence on the 0-50 GPa interval with the slope of $14.58 \mathrm{MHz} / \mathrm{GPa}$. On this smaller interval our results support the observed linear behavior and a restricted

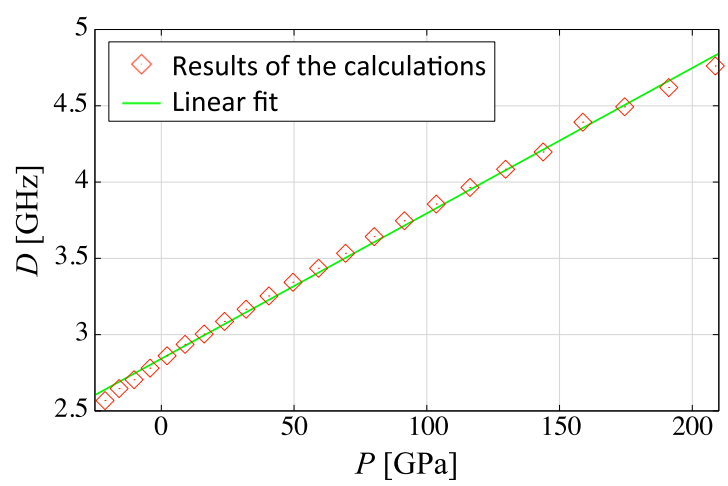

FIG. 3. (Color online) Calculated pressure dependence of parameter $D$ of the zero-field splitting tensor at zero temperature. The points show the calculated splitting at different ambient pressure applied on the defective supercell of the NV center in diamond. The line shows a linear fit with the slope of $9.52 \mathrm{MHz} / \mathrm{GPa}$. It is clearly seen that the calculated pressure dependence is mostly linear but it has a slight curvature on the examined large interval of the pressure. 
linear fit provides $10.30 \mathrm{MHz} / \mathrm{GPa}$ pressure dependence of the ZFS. This result is in fair agreement with the experimental data.

On the other hand, first-principles calculations allow us to carefully investigate macroscopic and microscopic effects, for example, in order to identify the most prominent contributions to the observed increment of the zero-field splitting. In our study we investigated four different sources of the variation of the ZFS. These effects can be divided up into two main classes as the matrix elements of the ZSFT depend on the distances of the spins and on the spatial distribution of the defect orbitals in a certain spin configuration. In the former case, there can be two main sources of the change of the distances, namely the macroscopic compression of the diamond lattice and the microscopic additional structural relaxation at the defect site. In the case of the NV center in diamond, the variation of the spatial distribution of the localized orbitals can be a consequence of the change of the localization of the electron density on different neighbor shells of the defect as well as the change of the shape of the individual localization at the atomic sites, for instance, through the variation of the $s p$ hybridization of the dangling bonds (Fig. 2 shows the localized spin density at the defect site).

In order to investigate the contribution of these effects, we have constructed different models and examined their response to the applied external pressure. In the case of the most complete nonsimplified first-principles model (model 1) we applied full geometry relaxation and a full self-consistent solution of the KS-particle system for several pressures. The results of these calculations were compared to the experimental measurements in the preceding paragraphs. In order to separate the effects of the macroscopic compression and the microscopic structural relaxation, we ignored the latter one in our second model (model 2). To achieve this we isotropically compressed the relaxed structure of the zero-pressure ground state and solved self-consistently the KS-particle system. Finally, to separate the effects of the change of the spatial distribution of the orbitals and the structure, in our third model (model 3) we omitted the self-consistent solution of the KS-particle system but simulated the compression of the supercell. This can be achieved analytically by replacing the defect orbital $\phi_{i}(\mathbf{r})$ with a spatially "scaled" and normalized one $N_{\alpha}^{-1 / 2} \phi_{i}\left(\alpha^{-1} \mathbf{r}\right)$, where $\alpha$ is a scaling factor, in the calculation of the matrix elements. The ZFS scales with $\alpha$ in accordance with the equation $D_{\alpha}=\alpha^{-3} D_{0}$, where $D_{0}$ is the zero-pressure value of the ZFS and the scaling factor $\alpha$ is either chosen to be the relative change of the lattice constant $\alpha_{a}(P)=a(P) / a_{0}$ or the relative change of the carbon-carbon distance in the first neighbor shell of the carbon vacancy $\alpha_{\mathrm{C}-\mathrm{C}}(P)=d_{\mathrm{C}-\mathrm{C}}(P) / d_{\mathrm{C}-\mathrm{C}}^{0}$. Model 3 , with the first definition of the scaling factor $\alpha$, can be considered as a further simplified version of model 2 , where the distance of the spins is in line with that of model 2 , i.e., only the macroscopic comparison is taken into account, however, here the wave function of the orbitals is not affected. With the second definition of $\alpha$, model 3 can be consider as a simplified version of model 1 , where the local relaxation is taken into account and the distance of the spin density localization in the first neighbor shell coincides with that of model 1.

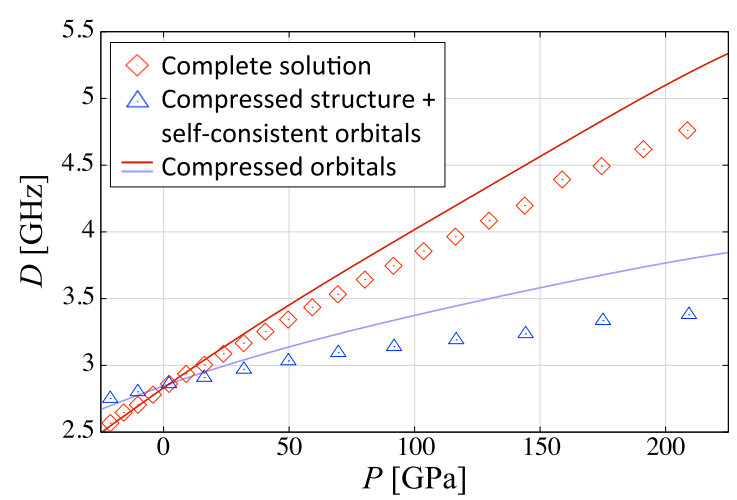

FIG. 4. (Color online) Calculated pressure dependence of parameter $D$ of the ZFS by using different models. The red points show the results of a complete self-consistent solution of the pressed supercells of a diamond embedding a single NV center (model 1). The blue triangles represent the results of a self-consistent solution of the KS system for the isotropically compressed supercell of the zero pressure structure (model 2). In this model the observed pressure dependence is a consequence of the macroscopic compression of the diamond lattice as well as the variation of the localization of the spin density. The blue (light gray) and red (dark gray) solid lines represent the zero-field splitting as calculated from analytical models (model 3) (see text for more details). The factor of compression $\alpha$ is in line with the pressure dependence of the lattice constant $\left[\alpha=a(P) / a_{0}\right]$ and with the pressure dependence of the $\mathrm{C}-\mathrm{C}$ distance in the first neighbor shell of the carbon vacancy $\left[\alpha=d_{\mathrm{C}-\mathrm{C}}(P) / d_{\mathrm{C}-\mathrm{C}}^{0}\right]$ in the case of blue (light gray) and red (dark gray) lines, respectively. In these models only the distance of the spins is affected, while the change of the spin density distribution is neglected.

The pressure dependence of the ZFS obtained from various models can be seen in Fig. 4. By comparing the results of model 1 and model 2 it can be concluded that the local structural relaxation has a large effect on the observed pressure dependence. Based on this comparison, one cannot decide on whether the local structural relaxation itself (the change of the distance of the spins) or the change in the wave functions adapted to the new position of the atoms is responsible for the additional shift. The relative position of the solid curves of the two different definitions of the analytic model (model 3) supports the importance of the local relaxation. On the other hand, in this model the change of the spatial distribution of the localized orbitals is neglected, therefore these results suggest that the change of the geometry, especially the geometry of the first neighbor shell, has the most prominent contribution to the pressure dependence. The changes of the distances of the nitrogen and carbon atoms in the first neighbor shell are presented in Table I. The distances of the carbon atoms are further decreased by a factor of 2 due to the local relaxation. This observation agrees with the results presented in Fig. 4 and therefore it highlights the importance of the local change of the structure. It can be also seen in Fig. 4 that the analytic model (when the wave functions are not adapted with the change of the positions of the atoms) is capable to reproduce well the result of the full self-consistent solution (model 1). This indicates that the inclusion of the variation of the orbitals has an opposite, but considerably smaller effect on the pressure dependence. 
TABLE I. Response of the microscopic structure of the NV center in diamond to applied external pressure. The distances between the nearest neighbors of the carbon vacancy $\mathrm{C}-\mathrm{C}$ and $\mathrm{C}-\mathrm{N}$ are presented for the cases of unpressed and pressed supercells. In the latter case, the effect of microscopic relaxation is highlighted by showing the results of compressed but fixed structure (model 2) and compressed and relaxed structure (model 1) calculations. The relative changes $(\Delta)$ with respect to the unpressed supercell are indicated in percentage.

\begin{tabular}{lcccc}
\hline \hline & $d_{\mathrm{C}-\mathrm{C}}(\AA)$ & $\Delta d_{\mathrm{C}-\mathrm{C}}$ & $d_{\mathrm{N}-\mathrm{C}}(\AA)$ & $\Delta d_{\mathrm{N}-\mathrm{C}}$ \\
\hline$P=0 \mathrm{GPa}$ & 2.660 & - & 2.729 & - \\
$P=209 \mathrm{GPa}$, model 2 & 2.420 & $-9.0 \%$ & 2.484 & $-9.0 \%$ \\
$P=209 \mathrm{GPa}$, model 1 & 2.176 & $-18.2 \%$ & 2.295 & $-15.9 \%$ \\
\hline \hline
\end{tabular}

Detailed analysis of the role of the variation of the spatial distribution of the spin density can be found in the Appendix. It turned out that the dangling bonds of the carbon atoms became more $s p^{3}$ hybridizedlike as a consequence of the local structural relaxation. Interestingly, this effect decreases the ZFS. The change of the spin density localization on the neighbor shells has only a minor effect.

To express our observations in numbers, we consider the 0-70 GPa interval where the pressure dependence is nearly linear. The effect of the bulk compression, the additional local structural relaxation at the defect site, and the additional variation of the spatial distribution of the spin density have $56 \%, 65 \%$, and $-21 \%$ contribution to the total pressure dependence, respectively. We depict all these contributions and compare with the experimental result in Fig. 5. Apparently the pressure dependence of the ZFS is governed by the distortion of the geometry and the change of the spatial distribution of the spin density has only a minor effect. Therefore, the second definition of model 3 , i.e., $D(P)=\alpha_{\mathrm{C}-\mathrm{C}}^{-3}(P) D_{0}$, can be a reasonable approximation in general. The observed slight nonlinearity of the pressure dependence on an extended interval is mainly the consequence of the inverse cubic dependence of $D(P)$ on $\alpha_{\mathrm{C}-\mathrm{C}}(P)$.

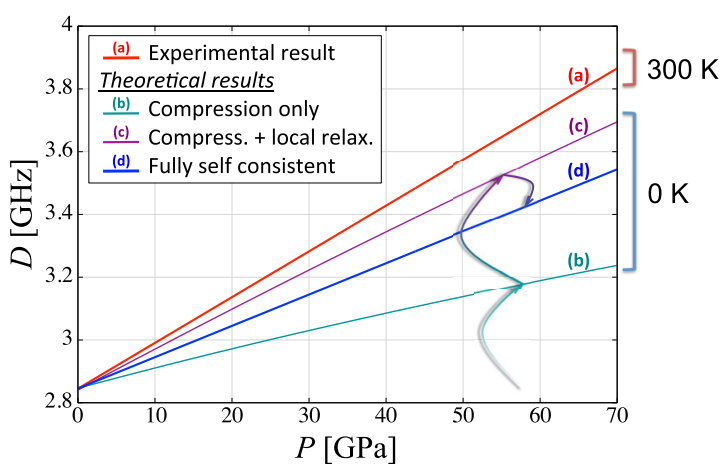

FIG. 5. (Color online) Comparison of the experimental result (a) with the results of different levels of theory (b), (c), and (d). In the most simplified method (b) only the effect of the macroscopic compression of the diamond lattice is taken into account. On the next level (c) the effect of the local structural relaxation is considered, however the wave functions are kept fixed. In the fully self-consistent solution (d) both the structure and the orbitals are relaxed (see text for more explanation).
Finally, we assess the similarities and the discrepancies of the present work and the literature. Recently, Doherty et al. proposed a theoretical model to account for the measured spin resonance shift induced by an applied external pressure [29]. In their study, they considered two effects: the structural distortion due to the compression of the bulk diamond lattice and the change of the spin density distribution on the neighbor shells of the NV center. The effects of the local structural relaxation at the defect site and the change of the $s p$ hybridization of the dangling bonds were neglected. First, they carried out a semiclassical calculation to determine the ZFS as a function of the structural compression. Based on the compressibility properties of the bulk diamond, 6.2 MHz/GPa pressure dependence of the ZFS is predicted at $P=0$, which accounts for $42.5 \%$ of the measured enhancement. Here we would like to mention that this model is equivalent to the $P \rightarrow 0$ limit of the first definition of our third model, where only the global compression is taken into account. We predict 6.26 MHz/GPa pressure dependence at $P=0$ by using the expression $D(P)=\left[\alpha_{a}(P)\right]^{-3} D_{0}$, with the experimental ZFS value $D_{0}$ and the $a b$ initio pressure dependent compression parameter $\alpha_{a}(P)=a(P) / a_{0}$ at $P=0$. This result is in excellent agreement with the semiclassical calculations [29]. On the other hand, the validity of our formula is extended and it predicts weak nonlinearity on larger pressure intervals. Doherty et al. attributed the remaining unexplained shift of the ZFS, $57.5 \%$ of the measured enhancement, to the change of the spin density distribution on the neighbor shells of the defect [29]. Our first-principles calculations allowed us to have a deeper insight and revealed that this effect has only a minor importance. The local structural relaxation is responsible for further important enhancement of the zero-field splitting as we pointed out above.

\section{B. Temperature dependence of zero-field splitting}

In this section we investigate the response of the ZFS to the thermal expansion of the bulk diamond. Additional effects, that are not included here, are the dynamical effects due to the thermal and zero-point motions as well as the effects of local anharmonicity.

The thermal expansion can be considered as the response of the macroscopic and microscopic structure to an applied negative hydrostatic pressure. From the preceding calculations, not just the pressure but the volume dependence of the ZFS can be determined as well. Since we studied isotropic compression and expansion of the defective supercell we could further determine the variation of the ZFS with respect to the relative change of the lattice constant $\eta=\Delta a / a_{0}$. A linear fit on the positive region, i.e., for expansion, yields $\theta=$ $d D / d \eta=-14.41 \mathrm{GHz}$ for the slope of the curve. In order to determine the temperature dependent shift of the ZFS one can use the identity $\Delta D(T)=\theta \eta(T)$, where $\eta(T)=\Delta a(T) / a_{0}=$ $\int_{0}^{T} \alpha\left(T^{\prime}\right) d T^{\prime}$, where $\alpha(T)$ is the linear thermal expansion coefficient that has been recently investigated experimentally by Stoupin et al. [38]. In a recent theoretical study it has been shown [39] that DFT simulations are capable to reproduce experimental data of $\alpha(T)$, thus we used the experimental parametrization of $\alpha(T)$. 


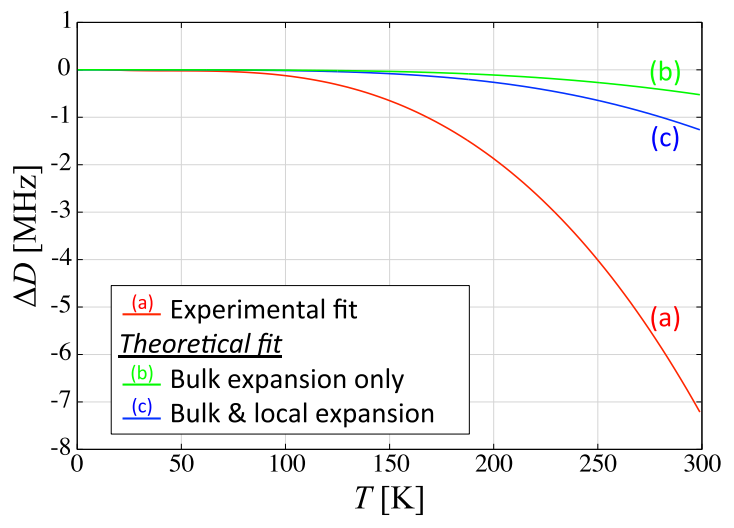

FIG. 6. (Color online) Experimental and theoretical temperature shifts of the zero-field splitting $\Delta D$ in the ground state of the $\mathrm{NV}$ center. Line (a) represents a fitted curve to the experimental measurements [15], while lines (b) and (c) show the theoretically predicted curves in the two models. In the case of line (b) only global expansion is taken into account, while in the case of line (c) both global expansion and local structural relaxation are adopted. The orbitals are treated self-consistently in both models.

The comparison of the experimental and theoretical results can be seen in Fig. 6. The outcomes of the simulations significantly deviate from the experiment, i.e., only $15 \%$ of the observed temperature shift is reproduced. Therefore, we conclude that a model, which includes thermal expansion only, cannot account for the temperature dependence of the ZFS. This conclusion is in good agreement with previous studies $[14,15,30]$. Furthermore, the predicted curve supports the results of the thermal expansion model of Doherty and co-workers [30].

Doherty et al. explained the remaining temperature shift of the ZFS by an effect of the electron-phonon coupling [30]. Based on the similarities of the temperature dependence of the optical zero-phonon lines (ZPLs) and the spin resonance [15], they introduced a unified treatment of these transitions to take into account contributions from the thermal expansion and the electron-phonon interaction. The latter contribution is assumed to be due to the different potential energy landscapes over the coordinate space in the excited and the ground states, which results in distinct phonon frequencies and thus in distinct thermal occupations of the phonon modes.

Our results confirm that inclusion of dynamical effects is required to obtain better description for the temperature dependence of the ZFS of the NV center.

\section{SUMMARY}

We demonstrated that $a b$ initio DFT theory calculations are able to account for the pressure dependence of the zero-field splitting of the NV center in diamond. We presented a detailed analysis of the microscopic and macroscopic effects and showed that the local structural deformations at the defect site plays a key role in the shift of the zero-field splitting. Our investigation was extended to the temperature dependence as well, where we found that the thermal expansion alone does not provide sufficient description to account for the observed decrease of the zero-field splitting at elevated temperatures.
Certainly dynamical effects, which is the subject of our ongoing research, can play an important role in this phenomena.

\section{ACKNOWLEDGMENTS}

Discussions with Professor Dmitry Budker is gratefully acknowledged. T.S., V.I., and A.G. acknowledge the support from the NIIF Supercomputer center Grant No. 1090, EU FP7 Grants No. 270197 (DIAMANT) and No. 611143 (DIADEMS), and the Lendület program of the Hungarian Academy of Sciences. V.I. and I.A.A. acknowledge support from Knut \& Alice Wallenberg Foundation "Isotopic Control for Ultimate Materials Properties" and Swedish National Infrastructure for Computing Grants No. SNIC 001/12-275 and No. SNIC 2013/1-331. J.R.M. acknowledges support from Conicyt (Chile) Grants Fondecyt No. 1141185, PIA ACT1108, and PIA ACT1112. I.A.A. is grateful to the Grant of Ministry of Education and Science of the Russian Federation (Grant No. 14.Y26.31.0005) and Tomsk State University Academic D. I. Mendeleev Fund Program.

\section{APPENDIX A: VARIATION OF THE SPATIAL DISTRIBUTION OF THE SPIN DENSITY}

In order to fully understand the importance of the variation of the spatial distribution of the defect orbitals, we analyzed the spin density localization on the neighbor shells of the NV center and the $s$ and $p$ decomposition of the dangling bonds (see Table II) and depicted the radial distribution of the total spin density in Fig. 7.

There are two main contributions to the change of the spatial distribution. One is the variation of the localization of the defect orbitals on the neighbor shells, i.e., they may become less or more expanded around the defect. The second one is the change of the shape of the localized spin density at the atomic sites. This is especially striking for the carbon dangling bonds, where most of the spin density is localized (see Fig. 2). First, we describe how the shape of these dangling bonds can change as a function of the pressure. The four atoms around the carbon vacancy in the nonperturbed ground state case relax outward

TABLE II. Response of the orbital localization of the NV center in diamond to external pressure. The relative spin density localization on the first neighbor shell of the carbon vacancy and the ratio of the $s$ - and $p$-orbital projected charge density of the dangling bonds of the carbon atoms (see Fig. 2. in the main text) are presented for the cases of unpressed and pressed supercells. To define the localization on the first neighbor shell we performed an integral of the spin density distribution in a sphere of scaled radius $r / d_{\text {cell }}=0.21$ centered on the carbon vacancy. In all cases, the effect of the microscopic relaxation is highlighted by showing the results of compressed but unrelaxed structure (model 2) and compressed and relaxed structure (model 1) calculations. The relative changes $(\Delta)$ with respect to the unpressed supercell are indicated in percentage.

\begin{tabular}{lcccc}
\hline \hline & $\varrho_{1}{ }^{\text {st }} / \varrho_{\text {tot }}$ & $\Delta\left(\varrho_{1}{ }^{\text {st }} / \varrho_{\text {tot }}\right)$ & $s / p$ & $\Delta(s / p)$ \\
\hline$P=0 \mathrm{GPa}$ & 0.793 & - & 0.0898 & - \\
$P=209 \mathrm{GPa}$, mod. 2 & 0.768 & $-3.15 \%$ & 0.0782 & $-12.9 \%$ \\
$P=209 \mathrm{GPa}$, mod. 1 & 0.798 & $+0.63 \%$ & 0.1695 & $+88.9 \%$ \\
\hline \hline
\end{tabular}




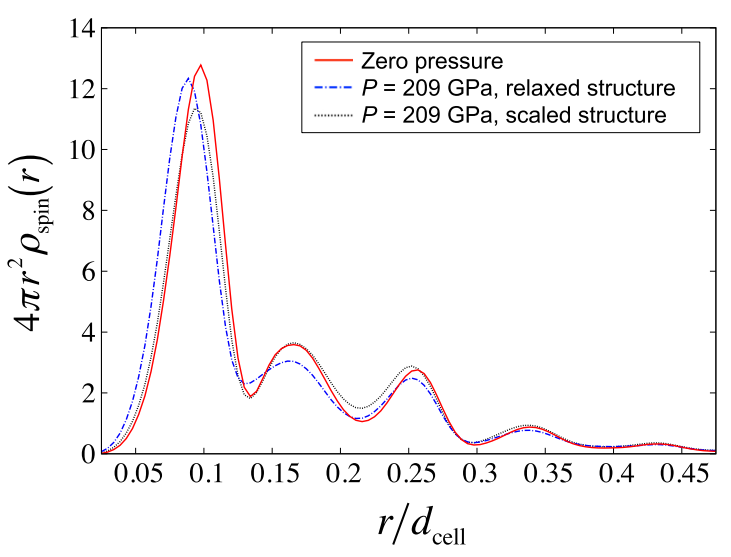

FIG. 7. (Color online) Angular integrated spin density distribution $\left[\rho_{\text {spin }}(r)\right]$ as a function of scaled radius $r / d_{\text {cell }}$, where $d_{\text {cell }}$ is the lattice constant of the supercell. The spherical coordinate system is centered on the carbon vacant site. The red solid line represents the spin density distribution of the unpressed supercell while the blue dot-dashed and the black dotted lines correspond to a pressed supercell ( $P=209 \mathrm{GPa}$ ) of models 1 and 2 , respectively. The first dip in the density distribution corresponds to the position of the first neighbor carbon atoms around the vacancy as labeled in the figure (see Fig. 2).

due to the broken bonds. As a consequence, the originally $s p^{3}$ hybridized bonds of these atoms become more $s p^{2}+p$ like and therefore the dangling bonds more $p$-like (see Fig. 2). Due to the compression and the local relaxation of the defect, the $s$ and $p$ decomposition of these dangling bond can change. The obtained hybridizations are presented in Table II for models 1 and 2. As one may notice, there is a remarkable increment in the $s$ contribution due to the local structural relaxation, however, the global compression slightly decreases it. The increment indicates more $s p^{3}$-like hybridization that agrees with the further decrease of the C-C distances, due to the local relaxation. In Fig. 7 the change of the $s / p$ ratio can be seen as the relative change of the area under the first and second peaks (around the first neighbor carbon atoms), while the change of the localization can be seen as the relative change of the area under the first two peaks and the area under the rest of the peaks. The changes of the $s / p$ ratio and the spin density localization in the different models, presented in Table II, can be clearly identified in Fig. 7.

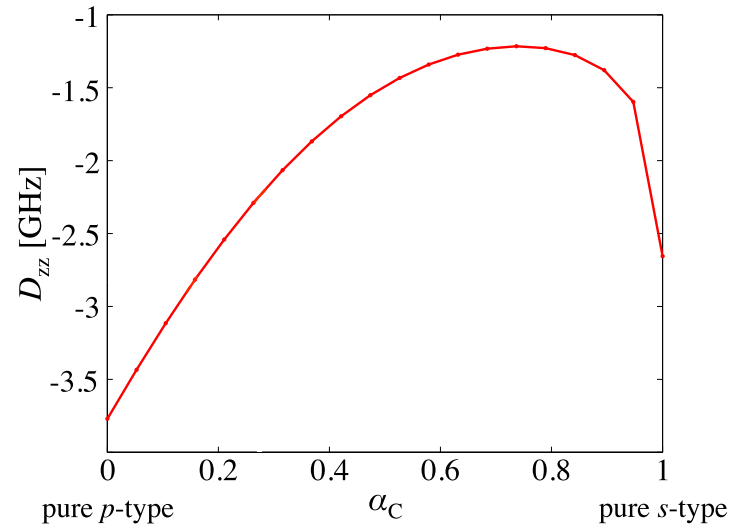

FIG. 8. (Color online) Matrix element of the zero-field splitting tensor as obtained from different model wave functions of linear combinations of $s$ and $p$ Gaussian orbitals. See text for the definition of the model wave function.

The contributions of these effects to the pressure dependence of the ZFS can be understood as follows. If the pressure causes increased (decreased) localization on the first neighbor carbon atoms around the vacancy, the value of the ZFS will enhance (decrease). As our results indicate, for the full self-consistent solution (model 1), this effect is small and thus can be neglected.

On the other hand, the effect of the variation of the $s / p$ ratio on the ZFS is not obvious. In order to investigate this effect, we modeled the dangling bonds of the carbon atoms $\sigma_{\mathrm{C}}$ with linear combination of Gaussian $s$ and $p$ orbitals,

$$
\sigma_{\mathrm{C}}=\alpha_{\mathrm{C}} \sigma_{s}+\sqrt{1-\alpha_{\mathrm{C}}^{2}} \sigma_{p},
$$

and calculated the $D_{z z}$ element of the ZFS tensor. Figure 8 shows that the more $s$-like orbitals were considered the smaller transition energy is obtained.

After these considerations, we can further understand the observed features showed in Fig. 5. For the full self-consistent model (model 1), the inclusion of the variation of the spatial distribution of the spin density decreases the ZFS due to the large increment of the $s / p$ ratio of the projected spin density of the carbon dangling bonds, while the effect of the small enhancement of the localization on the dangling bonds is negligible.
[1] L. du Preez, Ph.D. thesis, University of the Witwatersrand, Johannesburg, 1965.

[2] G. Davies and M. Hamer, Proc. R. Soc. London Ser. A 348, 285 (1976).

[3] G. Balasubramanian, I. Y. Chan, R. Kolesov, M. Al-Hmoud, J. Tisler, C. Shin, C. Kim, A. Wojcik, P. R. Hemmer, A. Krueger, T. Hanke, A. Leitenstorfer, R. Bratschitsch, F. Jelezko, and J. Wrachtrup, Nature (London) 455, 648 (2008).

[4] J. R. Maze, P. L. Stanwix, J. S. Hodges, S. Hong, J. M. Taylor, P. Cappellaro, L. Jiang, M. V. G. Dutt, E. Togan, A. S. Zibrov, A. Yacoby, R. L. Walsworth, and M. D. Lukin, Nature (London) 455, 644 (2008).
[5] V. M. Acosta, E. Bauch, M. P. Ledbetter, C. Santori, K.-M. C. Fu, P. E. Barclay, R. G. Beausoleil, H. Linget, J. F. Roch, F. Treussart, S. Chemerisov, W. Gawlik, and D. Budker, Phys. Rev. B 80, 115202 (2009).

[6] H. Mamin, M. Kim, M. Sherwood, C. Rettner, K. Ohno, D. Awschalom, and D. Rugar, Science 339, 557 (2013).

[7] T. Staudacher, F. Shi, S. Pezzagna, J. Meijer, J. Du, C. Meriles, F. Reinhard, and J. Wrachtrup, Science 339, 561 (2013).

[8] M. Grinolds, S. Hong, P. Maletinsky, L. Luan, M. Lukin, R. Walsworth, and A. Yacoby, Nat. Phys. 9, 215 (2013). 
[9] D. Le Sage, K. Arai, D. Glenn, S. DeVience, L. Pham, L. Rahn-Lee, M. Lukin, A. Yacoby, A. Komeili, and R. Walsworth, Nature (London) 496, 486 (2013).

[10] L. P. McGuinness, Y. Yan, A. Stacey, D. A. Simpson, L. T. Hall, D. Maclaurin, S. Prawer, P. Mulvaney, J. Wrachtrup, F. Caruso, R. E. Scholten, and L. C. L. Hollenberg, Nat. Nanotechnol. 6, 358 (2011).

[11] J. Wrachtrup and F. Jelezko, J. Phys. Condens. Matter 18, S807 (2006).

[12] L. Childress, M. V. Gurudev Dutt, J. M. Taylor, A. S. Zibrov, F. Jelezko, J. Wrachtrup, P. R. Hemmer, and M. D. Lukin, Science 314, 281 (2006).

[13] V. Jacques, P. Neumann, J. Beck, M. Markham, D. Twitchen, J. Meijer, F. Kaiser, G. Balasubramanian, F. Jelezko, and J. Wrachtrup, Phys. Rev. Lett. 102, 057403 (2009).

[14] V. M. Acosta, E. Bauch, M. P. Ledbetter, A. Waxman, L.-S. Bouchard, and D. Budker, Phys. Rev. Lett. 104, 070801 (2010).

[15] X.-D. Chen, C.-H. Dong, F.-W. Sun, C.-L. Zou, J.-M. Cui, Z.-F. Han, and G.-C. Guo, Appl. Phys. Lett. 99, 161903 (2011).

[16] D. M. Toyli, D. J. Christle, A. Alkauskas, B. B. Buckley, C. G. Van de Walle, and D. D. Awschalom, Phys. Rev. X 2, 031001 (2012).

[17] N. B. Manson, J. P. Harrison, and M. J. Sellars, Phys. Rev. B 74, 104303 (2006).

[18] J. R. Maze, A. Gali, E. Togan, Y. Chu, A. Trifonov, E. Kaxiras, and M. D. Lukin, New J. Phys. 13, 025025 (2011).

[19] M. W. Doherty, N. B. Manson, P. Delaney, and L. C. L. Hollenberg, New J. Phys. 13, 025019 (2011).

[20] A. S. Zyubin, A. M. Mebel, M. Hayashi, H. C. Chang, and S. H. Lin, J. Comput. Chem. 30, 119 (2009).

[21] Y. Ma, M. Rohlfing, and A. Gali, Phys. Rev. B 81, 041204 (2010).
[22] P. Delaney, J. C. Greer, and J. A. Larsson, Nano Lett. 10, 610 (2010).

[23] A. Ranjbar, M. Babamoradi, M. Heidari Saani, M. A. Vesaghi, K. Esfarjani, and Y. Kawazoe, Phys. Rev. B 84, 165212 (2011).

[24] S. K. Choi, M. Jain, and S. G. Louie, Phys. Rev. B 86, 041202 (2012).

[25] A. Gruber, A. Dräbenstedt, C. Tietz, L. Fleury, J. Wrachtrup, and C. v. Borczyskowski, Science 276, 2012 (1997).

[26] G. Kucsko, P. Maurer, N. Yao, M. Kubo, H. Noh, P. Lo, H. Park, and M. Lukin, Nature (London) 500, 54 (2013).

[27] P. Neumann, I. Jakobi, F. Dolde, C. Burk, R. Reuter, G. Waldherr, J. Honert, T. Wolf, A. Brunner, J. H. Shim, D. Suter, H. Sumiya, J. Isoya, and J. Wrachtrup, Nano Lett. 13, 2738 (2013).

[28] D. M. Toyli, F. Charles, D. J. Christle, V. V. Dobrovitski, and D. D. Awschalom, Proc. Natl. Acad. Sci. USA 110, 8417 (2013).

[29] M. W. Doherty, V. V. Struzhkin, D. A. Simpson, L. P. McGuinness, Y. Meng, A. Stacey, T. J. Karle, R. J. Hemley, N. B. Manson, L. C. L. Hollenberg, and S. Prawer, Phys. Rev. Lett. 112, 047601 (2014).

[30] M. W. Doherty, V. M. Acosta, A. Jarmola, M. S. Barson, N. B. Manson, D. Budker, and L. C. Hollenberg, Phys. Rev. B 90, 041201 (2014).

[31] P. E. Blöchl, Phys. Rev. B 50, 17953 (1994).

[32] G. Kresse and J. Furthmüller, Phys. Rev. B 54, 11169 (1996).

[33] J. P. Perdew, K. Burke, and M. Ernzerhof, Phys. Rev. Lett. 77, 3865 (1996).

[34] A. Gali, M. Fyta, and E. Kaxiras, Phys. Rev. B 77, 155206 (2008).

[35] A. Gali, Phys. Rev. B 80, 241204 (2009).

[36] M. J. Rayson and P. R. Briddon, Phys. Rev. B 77, 035119 (2008).

[37] Z. Bodrog and A. Gali, J. Phys. Condens. Matter 26, 015305 (2014).

[38] S. Stoupin and Y. V. Shvyd'ko, Phys. Rev. B 83, 104102 (2011).

[39] T. Ivanova and B. Mavrin, Phys. Solid State 55, 160 (2013). 